PROCEEDINGS OF THE

AMERICAN MATHEMATICAL SOCIETY

Volume 130, Number 6, Pages 1573-1580

S 0002-9939(01)06231-1

Article electronically published on October 24, 2001

\title{
THE HOMOGENEOUS SPECTRUM OF A GRADED COMMUTATIVE RING
}

\author{
WILLIAM HEINZER AND MOSHE ROITMAN
}

(Communicated by Wolmer V. Vasconcelos)

\begin{abstract}
Suppose $\Gamma$ is a torsion-free cancellative commutative monoid for which the group of quotients is finitely generated. We prove that the spectrum of a $\Gamma$-graded commutative ring is Noetherian if its homogeneous spectrum is Noetherian, thus answering a question of David Rush. Suppose $A$ is a commutative ring having Noetherian spectrum. We determine conditions in order that the monoid ring $A[\Gamma]$ have Noetherian spectrum. If $\operatorname{rank} \Gamma \leq 2$, we show that $A[\Gamma]$ has Noetherian spectrum, while for each $n \geq 3$ we establish existence of an example where the homogeneous spectrum of $A[\Gamma]$ is not Noetherian.
\end{abstract}

\section{INTRODUCTION}

All rings we consider are assumed to be nonzero, commutative and with unity. All the monoids are assumed to be torsion-free cancellative commutative monoids. Let $\Gamma$ be a monoid such that the group of quotients $G$ of $\Gamma$ is finitely generated, and let $R=\bigoplus_{\gamma \in \Gamma} R_{\gamma}$ be a commutative $\Gamma$-graded ring. A goal of this paper is to answer in the affirmative a question mentioned to one of us by David Rush as to whether $\operatorname{Spec} R$ is necessarily Noetherian provided the homogeneous spectrum, h-Spec $R$, is Noetherian.

If $I$ is an ideal of a ring $R$, we let $\operatorname{rad}(I)$ denote the radical of $I$, that is $\operatorname{rad}(I)=$ $\left\{r \in R: r^{n} \in I\right.$ for some positive integer $\left.n\right\}$. We say that $I$ is a radical ideal if $\operatorname{rad}(I)=I$. A subset $S$ of the ideal $I$ generates $I$ up to radical if $\operatorname{rad}(I)=\operatorname{rad}(S R)$. The ideal $I$ is radically finite if it is generated up to radical by a finite set.

We recall that a ring $R$ is said to have Noetherian spectrum if the set $\operatorname{Spec} R$ of prime ideals of $R$ with the Zariski topology satisfies the descending chain condition on closed subsets. In ideal-theoretic terminology, $R$ has Noetherian spectrum if and only if $R$ satisfies the ascending chain condition (a.c.c.) on radical ideals. Thus a Noetherian ring has Noetherian spectrum and each ring having only finitely many prime ideals has Noetherian spectrum. As is shown in [8, Prop. 2.1], Spec $R$ is Noetherian if and only if each ideal of $R$ is radically finite. It is well known that $R$ has Noetherian spectrum if and only if $R$ satisfies the two properties: (i) a.c.c. on prime ideals, and (ii) every ideal of $R$ has only finitely many minimal prime ideals [6], 3. Theorem 88, page 59 and Ex. 25, page 65].

Received by the editors September 20, 2000 and, in revised form, December 13, 2000.

1991 Mathematics Subject Classification. Primary 13A15, 13 E99.

Key words and phrases. Graded ring, homogeneous spectrum, Noetherian spectrum, torsionfree cancellative commutative monoid.

This work was prepared while the second author enjoyed the hospitality of Purdue University. 
In analogy with the result of Cohen that a ring $R$ is Noetherian if each prime ideal of $R$ is finitely generated, it is shown in [8, Corollary 2.4] that $R$ has Noetherian spectrum if each prime ideal of $R$ is radically finite. It is shown in 8 Theorem 2.5] that Noetherian spectrum is preserved under polynomial extension in finitely many indeterminates. Thus finitely generated algebras over a ring with Noetherian spectrum again have Noetherian spectrum.

In Section 1 we prove that if $R$ is a $\Gamma$-graded ring, where $\Gamma$ is a monoid with finitely generated group of quotients, and if h-Spec $R$ is Noetherian, then $\operatorname{Spec} R$ is Noetherian (Theorem 1.7). In Section 2 we deal with monoid rings. It turns out that if $M$ is a monoid with finitely generated group of quotients and $k$ is a field, then the homogeneous spectrum of the monoid ring $k[M]$ is not necessarily Noetherian (Example 2.9). On the positive side, h-Spec $A[M]$ is Noetherian if $A$ is a ring with Noetherian spectrum and $M$ is a monoid of torsion-free rank $\leq 2$.

We thank David Rush for pointing out several errors in an earlier version of this paper.

\section{The homogeneous SPECTRUM}

The homogeneous spectrum, h-Spec $R$, of a graded ring $R=\bigoplus_{\gamma \in \Gamma} R_{\gamma}$ is the set of homogeneous prime ideals of $R$. The most common choices for the commutative monoid $\Gamma$ are the monoid $\mathbb{N}$ of nonnegative integers or its group of quotients $\mathbb{Z}$. A standard technique using homogeneous localization shows the following: if $R=$ $\bigoplus_{n \in \mathbb{Z}} R_{n}$ is a $\mathbb{Z}$-graded integral domain, if $t$ is a nonzero element of $R_{1}$, and if $H$ is the multiplicative set of nonzero homogeneous elements of $R$, then the localization $R_{H}$ of $R$ with respect to $H$ is the graded Laurent polynomial ring $K_{0}\left[t, t^{-1}\right]$, where $K_{0}$ is a field [10 page 157]. This implies the following remark.

Remark 1.1. Suppose $R=\bigoplus_{n \in \mathbb{Z}} R_{n}$ is a graded integral domain and $P$ is a nonzero prime ideal of $R$. If zero is the only homogeneous element contained in $P$, then the localization $R_{P}$ is one-dimensional and Noetherian.

If $R=\bigoplus_{n \in \mathbb{Z}} R_{n}$ is a graded ring with no nonzero homogeneous prime ideals, then $R_{0}$ is a field and either $R=R_{0}$, or $R$ is a Laurent polynomial ring $R_{0}\left[x, x^{-1}\right]$ [1, page 83].

Every ring can be viewed as a graded ring with the trivial gradation that assigns degree zero to every element of the ring. Thus Nagata in [7) Section 8] develops primary decomposition for graded ideals in a graded Noetherian ring. It is not surprising that there is an interrelationship among the Noetherian properties of $\operatorname{Spec} R, \mathrm{~h}-\operatorname{Spec} R$, Spec $R[X]$ and h-Spec $R[X]$.

Proposition 1.2 is useful in considering the Noetherian property of spectra. It follows by induction from [8, Prop. 2.2 (ii)], but we prefer to prove it directly.

Proposition 1.2. Let $I$ be an ideal of a ring $R$. Let $J$ be an ideal of $R$ and $S$ a subset of $J$ such that $J=\operatorname{rad} S R$. If $I+J$ is radically finite and if for each $s \in S$ the ideal $I R[1 / s]$ is radically finite, then $I$ is radically finite.

Proof. Since $I+J$ is radically finite and since $J=\operatorname{rad} S R$, there exist finite sets $F \subseteq I$ and $G \subseteq S$ such that $\operatorname{rad}(I+J)=\operatorname{rad}((F, G) R)$. For each $g \in G$ there exists a finite subset $T_{g}$ of $I$ such that $\operatorname{rad}(I R[1 / g])=\operatorname{rad}\left(T_{g} R[1 / g]\right)$. Let $I^{\prime}=$ $\left(F \cup \bigcup_{g \in G} T_{g}\right) R$, thus $I^{\prime} \subseteq I$. Suppose $P \in \operatorname{Spec} R$ and $I^{\prime} \subseteq P$. If $G \subseteq P$, then $I \subseteq P$ since $\operatorname{rad}\left(I^{\prime}+G R\right)=\operatorname{rad}(I+J)$. Otherwise, we have $g \notin P$ for some element $g \in G$. Therefore $\operatorname{rad}\left(I^{\prime} R[1 / g]\right)=\operatorname{rad}(I R[1 / g]) \subseteq P R[1 / g]$. Since $P$ is the 
preimage in $R$ of $P R[1 / g]$, we have $\operatorname{rad}(I) \subseteq P$. Therefore $\operatorname{rad}\left(I^{\prime}\right)=\operatorname{rad}(I)$, so $I$ is radically finite.

For Corollary 1.3, we use that the (homogeneous) spectrum of a graded ring $R$ is Noetherian iff each (homogeneous) ideal of $R$ is radically finite.

Corollary 1.3. (1) Let $S$ be a finite subset of a ring $R$. If $\operatorname{Spec}(R / S R)$ is Noetherian and for each $s \in S$, $\operatorname{Spec}(R[1 / s])$ is Noetherian, then $\operatorname{Spec} R$ is Noetherian.

(2) Let $S$ be a finite set of homogeneous elements of a graded ring $R$. If $\mathrm{h}-\operatorname{Spec}(R / S R)$ is Noetherian and for each $s \in S$, h-Spec $(R[1 / s])$ is Noetherian, then h-Spec $R$ is Noetherian.

The hypotheses in Proposition 1.2 and Corollary 1.3 concerning the set $S$ may be modified as follows and still give the same conclusion:

Proposition 1.4. Let $I$ be an ideal of a ring $R$, and let $S$ be a finite subset of $R$. Let $U$ be the multiplicatively closed subset of $R$ generated by $S$.

(1) If $I+s R$ is radically finite for each $s \in S$ and $I R_{U}$ is radically finite, then $I$ is radically finite.

(2) If $\operatorname{Spec}(R / s R)$ is Noetherian for each $s \in S$ and $\operatorname{Spec} R_{U}$ is Noetherian, then $\operatorname{Spec} R$ is Noetherian.

(3) If $R$ is a $\Gamma$-graded ring for some monoid $\Gamma$, each $s \in S$ is homogeneous with $\mathrm{h}-\operatorname{Spec}(R / s R)$ Noetherian and if h-Spec $R_{U}$ is Noetherian, then h-Spec $R$ is Noetherian.

The next corollary is a special case of Proposition 1.2.

Corollary 1.5. Suppose $S$ is a subset of a ring $R$ that generates $R$ as an ideal and let $I$ be an ideal of $R$. If $I R[1 / s]$ is radically finite for each $s \in S$, then $I$ is radically finite.

If $R[1 / s]$ has Noetherian spectrum for each $s \in S$, then $R$ has Noetherian spectrum.

In analogy with Corollary 1.5, it is a standard result in commutative algebra that if $S R=R$ and $R[1 / s]$ is a Noetherian ring for each $s \in S$, then $R$ is a Noetherian ring. However, the analogue of Corollary 1.3 for the Noetherian property of a ring is false: There exists a non-Noetherian ring $R$ and an element $s \in R$ such that $R / s R$ and $R[1 / s]$ are Noetherian. For example, let $X, Y$ be indeterminates over a field $k$, let $R:=k\left[X,\left\{Y / X^{n}\right\}_{n=0}^{\infty}\right]$ and let $s=X$. Then $P=\left(\left\{Y / X^{n}\right\}_{n=0}^{\infty}\right)$ is a nonfinitely generated prime ideal of $R$, so $R$ is not Noetherian, although both $R / X R=k$ and $R[1 / X]=k[X, Y, 1 / X]$ are Noetherian. Incidentally, both the ideal $(P+X R) / X R=(0)$ of $R / X R$ and the ideal $P R[1 / X]$ of $R[1 / X]$ are principal.

Proposition 1.6 is the graded analogue of [8, Theorem 2.5].

Proposition 1.6. Suppose $\Gamma$ is a torsion-free cancellative commutative monoid with a group of quotients $G$ and $R=\bigoplus_{\gamma \in \Gamma} R_{\gamma}$ is a $\Gamma$-graded commutative ring. Fix $g \in G$, and consider the polynomial ring $R[X]$ as a graded extension ring of $R$ uniquely determined by defining $X$ to be a homogeneous element of degree $g$. If h-Spec $R$ is Noetherian, then h-Spec $R[X]$ is Noetherian.

Proof. Assume that h-Spec $R$ is Noetherian, but h-Spec $R[X]$ is not Noetherian. Then there exists a homogeneous prime ideal $P$ of $R[X]$ that is maximal with respect 
to not being radically finite. Since $P \cap R=p$ is a homogeneous prime ideal of $R$ and h-Spec $R$ is Noetherian, we may pass from $R[X]$ to $R[X] / p[X] \cong(R / p)[X]$ and assume that $P \cap R=(0)$. Then $R$ is a graded domain and h-Spec $R$ is Noetherian. Choose an element $f \in P$ having minimal degree $d$ as a polynomial in $R[X]$. By replacing $f$ by one of its nonzero homogeneous components, we may assume that $f=a_{d} X^{d}+a_{d-1} X^{d-1}+\cdots+a_{0}$, where the elements $a_{i} \in R$ are homogeneous elements of $R$ with $a_{d} \neq 0$. Since $P \cap R=(0)$, we have $d>0$ and $a_{d} \notin P$. The maximality of $P$ with respect to not being radically finite implies $\left(P, a_{d}\right) R[X]$ is radically finite. Since $a_{d}^{-1} f$ is a polynomial of minimal degree in $P R\left[1 / a_{d}\right][X]$ and since this polynomial is monic in $R\left[1 / a_{d}\right][X]$, we see that $P R\left[1 / a_{d}\right][X]=(f)$. But Proposition 1.4 (1) then implies that $P$ is radically finite, a contradiction.

We use Proposition 1.6 in the proof of Theorem 1.7

Theorem 1.7. Let $R=\bigoplus_{\gamma \in \Gamma} R_{\gamma}$ be a $\Gamma$-graded commutative ring, where $\Gamma$ is a nonzero torsion-free cancellative commutative monoid such that its group of quotients $G$ is finitely generated. If h-Spec $R$ is Noetherian, then $\operatorname{Spec} R$ is also Noetherian.

Proof. Up to a group isomorphism, we have $G \cong \mathbb{Z}^{d}$ for some positive integer $d$. Hence we may assume $G=\mathbb{Z}^{d}$. For $1 \leq i \leq d$, let $g_{i}$ be the element of $G$ having 1 as its $i$-th coordinate and zeros elsewhere. Consider the graded polynomial extension ring $R[\mathbf{X}]:=R\left[X_{1}, \ldots, X_{d}\right]$ obtained by defining $X_{i}$ to be a homogeneous element of degree $g_{i}$ for $i=1, \ldots, d$. Proposition 1.6 implies that h-Spec $R[\mathbf{X}]$ is Noetherian. We associate with each nonzero element $r \in R$ a homogeneous element $\widetilde{r} \in R[\mathbf{X}]$ such that $\operatorname{deg}(\widetilde{r})=\left(c_{1}, \ldots, c_{d}\right)$, where $c_{j}$ is the maximum of the $j$-th coordinates of the degrees of the nonzero homogeneous components of $r \in R$ as follows: let $r=r_{1}+\cdots+r_{k}$ be the homogeneous decomposition of $r$; set $\widetilde{r}=\sum_{i=1}^{k} r_{i} \mathbf{X}^{m_{i}}$, where $m_{i}=\left(c_{1}, \ldots, c_{d}\right)-\operatorname{deg} r_{i}$ for each $i$ and $\mathbf{X}^{\left(a_{1}, \ldots, a_{d}\right)}=\prod_{i=1}^{d} X_{i}^{a_{i}}$ for each sequence $\left(a_{1}, \ldots, a_{d}\right)$ in $\mathbb{Z}^{d}$. We define $\widetilde{0}=0$. With each ideal $I$ of $R$, let $\widetilde{I}$ denote the homogeneous ideal of $R[\mathbf{X}]$ generated by $\{\widetilde{r}: r \in I\}$ ( $\widetilde{r}$ is the homogenization of $r$ and $\widetilde{I}$ is the homogenization of $I$ ).

Let $\phi: R[\mathbf{X}] \rightarrow R$ denote the $R$-algebra homomorphism defined by $\phi\left(X_{i}\right)=1$ for $i=1, \ldots, n$. Since $\phi$ is an $R$-algebra homomorphism and $\phi(\widetilde{r})=r$ for each $r \in R$, for each ideal $I$ of $R$, we have $\phi(\widetilde{I})=I$ (the meaning of $\phi$ is dehomogenization). Therefore the map $I \rightarrow \widetilde{I}$ is a one-to-one inclusion preserving correspondence of the set of ideals of $R$ into the set of homogeneous ideals of $R[\mathbf{X}]$.

Let $I$ be an ideal of $R$. Since h-Spec $R[\mathbf{X}]$ is Noetherian there exists a finite set $S$ such that $\operatorname{rad} \widetilde{I}=\operatorname{rad}(S R[\mathbf{X}])$. We have $\operatorname{rad} I=\operatorname{rad} \phi(\widetilde{I})=\operatorname{rad}(\phi(S)) R$, thus $I$ is radically finite. Therefore $\operatorname{Spec} R$ is Noetherian.

The following corollary is immediate from Theorem 1.7

Corollary 1.8. Let $R$ be an $\mathbb{N}$-graded or a $\mathbb{Z}$-graded ring. If h-Spec $R$ is Noetherian, then $\operatorname{Spec} R$ is Noetherian.

Without the assumption in Theorem 1.7 that the group of quotients of $\Gamma$ is finitely generated, it is possible to have h-Spec $R$ be Noetherian and yet $\operatorname{Spec} R$ not be Noetherian. For example, if $K$ is an algebraically closed field of characteristic zero and $\Gamma=\mathbb{Q}$, then $(0)$ is the only homogeneous prime ideal of the group ring $R:=K[\mathbb{Q}]$ so h-Spec $R$ is Noetherian, but as we note in Theorem [2.6 below, Spec $R$ is not Noetherian. 


\section{The NoETherian SPECTRA OF MONOID RINGS}

Suppose $A$ is a ring and $M$ is a cancellative torsion-free commutative monoid. We consider the monoid ring $A[M]$ as a graded ring with its natural $M$-grading where the nonzero elements of $A$ are of degree zero. The monoid $M$ is naturally identified with a subset of $A[M]$. We write $X^{m}$ for $m \in M \subseteq A[M]$. Note that $0 \in M$ is identified with $1 \in A[M]$.

A $\mathbb{Q}$-monoid in a $\mathbb{Q}$-vector space $V$ is an additive submonoid of $V$ that is closed under multiplication by positive rationals. A subset of a $\mathbb{Q}$-monoid $W$ is called a $\mathbb{Q}$-ideal of the $\mathbb{Q}$-monoid $W$ if it is an ideal of the monoid $W$ that is closed under multiplication by positive (that is, strictly positive) rationals.

If $M$ is a cancellative torsion-free monoid with group of quotients $G$, we denote by $M^{(\mathbb{Q})}$ the $\mathbb{Q}$-monoid generated by $M$ in $G \otimes_{\mathbb{Z}} \mathbb{Q}$; thus $M=\{q m \mid q>0$ in $\mathbb{Q}, m \in$ $M\}$.

Remark 2.1. Let $S$ be a subset of a monoid $M$, let $R$ be a ring, and let $I$ be a homogeneous ideal of $R[M]$ containing $S$ and generated by monomials in $M$. Then $S$ generates $I$ up to radical iff $S$ generates the $\mathbb{Q}$-ideal $(I \cap M)^{(\mathbb{Q})}$ of $M^{(\mathbb{Q})}$.

Remark 2.2. Suppose $M$ is a cancellative torsion-free commutative monoid and $k$ is a field. There is a natural one-to-one inclusion preserving correspondence between the homogeneous radical ideals of the monoid domain $k[M]$ and the $\mathbb{Q}$-ideals of the $\mathbb{Q}$-monoid $M^{(\mathbb{Q})}$. Indeed, to each $\mathbb{Q}$-ideal $L$ of $M^{(\mathbb{Q})}$ (which is generated by $L \cap M$ ) we make correspond the ideal of $k[M]$ generated by $L \cap M$.

Lemma 2.3. Suppose $M$ is a torsion-free cancellative commutative monoid, $A$ is a ring with Noetherian spectrum, and $P$ is a homogeneous prime ideal of the monoid ring $A[M]$. Then the following two conditions are equivalent:

(1) The prime ideal $P$ is radically finite in $A[M]$.

(2) The $\mathbb{Q}$-ideal $(P \cap M)^{(\mathbb{Q})}$ of $M^{(\mathbb{Q})}$ is finitely generated.

Proof. Since $P$ is prime and homogeneous, it is generated by $(P \cap A) \cup(P \cap M)$. Since $\operatorname{Spec} A$ is Noetherian, we see that $P$ is radically finite iff the ideal in $A[M]$ generated by $P \cap M$ is radically finite iff the $\mathbb{Q}$-ideal $(P \cap M)^{(\mathbb{Q})}$ of $M^{(\mathbb{Q})}$ is finitely generated (Remark 2.1). This proves Lemma 2.3.

The following is an immediate corollary to Lemma [2.3.

Corollary 2.4. Let $M$ be a torsion-free cancellative commutative monoid and let $A$ be a ring with Noetherian spectrum. Then the following two conditions are equivalent:

(1) The monoid ring $A[M]$ has Noetherian homogeneous spectrum.

(2) Each $\mathbb{Q}$-ideal in the $\mathbb{Q}$-monoid $M^{(\mathbb{Q})}$ is finitely generated.

We denote the torsion-free rank of a monoid $M$ by $\operatorname{rank} M$.

Proposition 2.5. Suppose $A$ is a ring and $M$ is a cancellative torsion-free commutative monoid.

(1) If $\operatorname{Spec} A[M]$ is Noetherian, then $\operatorname{Spec} A$ is Noetherian and $\operatorname{rank} M$ is finite.

(2) If $\operatorname{Spec} A$ is Noetherian and if $\operatorname{rank} M \leq 2$, then $\mathrm{h}-\operatorname{Spec} A[M]$ is Noetherian.

Proof. (1) Spec $A$ is Noetherian since every ideal $I$ of $A$ satisfies $I=I A[M] \cap A$, and if $I$ is a radical ideal of $A$, then $I A[M]$ is a radical in $A[M]$. On the other hand, 
if $\operatorname{rank} M$ is infinite, let $B$ be an infinite set of elements in $M$ which are linearly independent over $\mathbb{Q}$ in the $\mathbb{Q}$-vector space $G \otimes_{\mathbb{Z}} \mathbb{Q}$, where $G$ is the group of quotients of $M$. Then the ideal of $A[M]$ generated by the elements $X^{b}-1$ for $b \in B$ is not radically finite. Therefore h-Spec $A[M]$ is not Noetherian.

(2) By Lemma 2.3 it suffices to show that each $\mathbb{Q}$-ideal in $M^{(\mathbb{Q})}$ is finitely generated. We may assume that $M \subseteq \mathbb{Q}^{2}$. Let $W$ be a nonempty $\mathbb{Q}$-ideal of $M^{(\mathbb{Q})}$. We show that $W$ is a finitely generated ideal of $\widetilde{W}:=W \cup\{\mathbf{0}\}$. If $W$ spans a one-dimensional subspace and $\mathbf{v}$ is a nonzero element of $W$, then the $\mathbb{Q}$-ideal $W$ is generated by $\mathbf{0}$ if $-\mathbf{v} \in W$, and by $\mathbf{v}$ otherwise. If $W$ spans $\mathbb{Q}^{2}$, then choose two linearly independent vectors in $W$. By changing coordinates, we may assume that these vectors are $(1,0)$ and $(0,1)$. If $W$ contains a vector $\mathbf{v}$ with both coordinates strictly negative, then $W$ is generated by $\mathbf{0}$ as a $\mathbb{Q}$-ideal. Otherwise, define vectors $\mathbf{u}$ and $\mathbf{v}$ as follows: if $a=\min \{y \mid(1, y) \in W\}$ exists, let $\mathbf{u}=(1, a)$; if the minimum does not exist, let $\mathbf{u}=(1,0)$. Similarly, define a vector $\mathbf{v}$ with second coordinate 1 . Then $\mathbf{u}$ and $\mathbf{v}$ generate $W$ as a $\mathbb{Q}$-ideal of $\widetilde{W}$.

Theorem 2.6. Let $A$ be a ring with Noetherian spectrum and $M$ be a cancellative torsion-free commutative monoid. If the group of quotients of $M$ is finitely generated and if $\operatorname{rank} M \leq 2$, then the monoid ring $A[M]$ has Noetherian spectrum.

On the other hand, if $A[M]$ has Noetherian spectrum and if $A$ contains an algebraically closed field of zero characteristic, then the group of quotients of $M$ is finitely generated.

Proof. Assume that the group of quotients of $M$ is finitely generated and that $\operatorname{rank} M \leq 2$. By Proposition [2.5 (2), $A[M]$ has Noetherian homogeneous spectrum. By Theorem 1.7, Spec $A[M]$ is Noetherian.

For the second statement, assume that the group of quotients of $M$ is not finitely generated. By Proposition 2.5 (1), we may assume that $M$ has finite rank. It follows that there exists an element $s \in M$ that is divisible by infinitely many positive integers. Since $A$ contains all roots of unity and they are distinct, we obtain that over the element $X^{s}-1$ of $A[M]$ there are infinitely many minimal primes. Therefore $\operatorname{Spec} A[M]$ is not Noetherian.

With regard to Theorem 2.6 if the monoid $M$ is finitely generated, then it follows from [8, Theorem 2.5], that $\operatorname{Spec} A[M]$ is Noetherian if $\operatorname{Spec} A$ is Noetherian.

Example 2.7. Over a field $k$ of characteristic $p>0$, there exists a monoid $M$ for which the group of quotients is not finitely generated and yet the monoid domain $k[M]$ has Noetherian spectrum. For example, if $M:=\mathbb{Z}\left[\left\{1 / p^{n}\right\}_{n=1}^{\infty}\right]$, then $k[M]$ is an integral purely inseparable extension of $k[\mathbb{Z}]$ and $\operatorname{Spec}(k[M])$ is Noetherian.

A prime $\mathbb{Q}$-ideal of a $\mathbb{Q}$-monoid $M$ is a $\mathbb{Q}$-ideal $Q$ of $M$ that is a prime ideal, that is, if $a+b \in Q$, then either $a \in Q$ or $b \in Q$.

Let $S$ be a subset of a vector space over $\mathbb{Q}$. $S$ is $\mathbb{Q}$-convex if for any points $p, q$ in $S$ and rational $0 \leq t \leq 1$ we have $t p+(1-t) q \in S$.

Remark 2.8. Let $M$ be a $\mathbb{Q}$-monoid in a vector space over $\mathbb{Q}$, and let $I$ be a subset of $M$ that is closed under addition and under multiplication by positive rationals; thus $I$ is a $\mathbb{Q}$-convex set. Then $I$ is an ideal of $M$ iff for any two points $p \in I$ and $q \in M$ and any rational $0<t<1$, we have $t p+(1-t) q \in I$. Moreover, for $I$ as above, if $I$ is an ideal, then $I$ is prime iff the set $M \backslash I$ is $\mathbb{Q}$-convex. 
We denote by $C$ the unit circle in $\mathbb{R}^{2}$, that is, $C=\left\{(x, y) \in \mathbb{R}^{2}: x^{2}+y^{2}=1\right\}$. We let $C_{\mathbb{Q}}=C \cap \mathbb{Q}^{2}$. For any subset $S$ of $\mathbb{R}^{n}$ we denote by $S^{+}$the set of points in $S$ with nonnegative coordinates.

Example 2.9. Let $n \geq 3$. Then there exists a cancellative torsion-free commutative monoid $M$ of rank $n$ such that the group of quotients of $M$ is finitely generated, but for any ring $A$ the homogeneous spectrum of $A[M]$ is not Noetherian. Furthermore, the monoid $M$ is completely integrally closed. Hence, if $A$ is an integrally closed (completely integrally closed) domain, then $A[M]$ is an integrally closed (completely integrally closed) domain.

First let $n=3$. Let $W$ be the $\mathbb{Q}$-submonoid of $\mathbb{Q}^{3}$ generated by the set $\left\{(x, y, 1):(x, y) \in C_{\mathbb{Q}}\right\}$. We claim that the $\mathbb{Q}$-ideal $W \backslash\{\mathbf{0}\}$ of $W$ is not finitely generated; moreover, if $(p, 1) \in C_{\mathbb{Q}} \times\{1\}$, then $(p, 1)$ does not belong to the $\mathbb{Q}$-ideal of $W$ generated by $C_{\mathbb{Q}} \times\{1\} \backslash\{(p, 1)\}$. Indeed, by Remark 2.8, the set of points $(x, y, z) \in W$ such that $\frac{1}{z}(x, y) \neq p$ is a $\mathbb{Q}$-ideal of $W$ which does not contain $(p, 1)$. Set $M=W \cap \mathbb{Z}^{3}$. More explicitly, since the convex hull of $C_{\mathbb{Q}}$ equals the rational unit disk, we see that $M=\left\{X^{a} Y^{b} Z^{c} \mid(a, b, c) \in \mathbb{Z}^{3}, c \geq 0\right.$ and $\left.a^{2}+b^{2} \leq c^{2}\right\}$.

Now let $A$ be any ring. Since the $\mathbb{Q}$-ideal generated by $W \backslash\{\mathbf{0}\}$ in $W$ is not finitely generated, we obtain by Lemma 2.3 that the ideal in $A[M]$ generated by the nonzero elements of $M$ is not radically finite; thus h-Spec $A[M]$ is not Noetherian.

If $n>3$ let $\widetilde{M}=M \oplus \mathbb{Z}^{n-3}$, where $M$ is the monoid defined above. Then $\operatorname{rank} \widetilde{M}=n$ and $\widetilde{M}$ satisfies our requirements.

Clearly, $M$ is a completely integrally closed monoid. Thus the assertions on $A[M]$ follow from [2, Corollary 12.7 (2) and Corollary 12.11 (2)].

We now elaborate on Example 2.9, but with $W$ replaced by $W^{+}$. As seen in Example [2.9, $R$ is a completely integrally closed domain, and h-Spec $R$ is not Noetherian. Moreover, $R=k[M]$ is a subring of the polynomial ring $k[X, Y, Z]$ and has fraction field $k(X, Y, Z)$. By [2, Theorem 21.4], $\operatorname{dim} R=3$. It is interesting that the maximal homogeneous ideal $N$ of $R$ has height 3 , but its homogeneous height (defined using just homogeneous prime ideals) is 2 . Indeed, let $P \neq N$ be a nonzero prime homogeneous ideal of $R$. Let $Q$ be the $\mathbb{Q}$-ideal of $W$ generated by the points $(a, b, c)$ in $\mathbb{Q}^{3}$ such that $X^{a} Y^{b} Z^{c} \in P$. Since $Q$ is a prime $\mathbb{Q}$-ideal of $W$ and since $C_{\mathbb{Q}}^{+}$is dense in $C^{+}$, by Remark 2.8 we easily obtain that $Q$ contains $C_{\mathbb{Q}}^{+} \times\{1\}$ except one point. Thus the homogeneous height of $N$ is at most 2. Since the $\mathbb{Q}$-ideal of $W$ generated by $C_{\mathbb{Q}}^{+} \times\{1\}$ with one point removed is prime, we see that the homogeneous height of $N$ is 2 .

On the other hand, ht $N=3$. More generally, if $R$ is a $k$-subalgebra of a polynomial ring $k[\mathbf{X}]:=k\left[X_{1}, \ldots, X_{n}\right]$ over a field $k$ with the same fraction field $k(\mathbf{X})$, then $\operatorname{ht}((\mathbf{X}) k[\mathbf{X}] \cap R)=n$. Indeed, this prime ideal has height at most $n$ since $k(\mathbf{X})$ has transcendence degree $n$ over $k$. Moreover, each nonzero ideal of $k[\mathbf{X}]$ has a nonzero intersection with $R$. Since the primes of height $n-1$ of $k[\mathbf{X}]$ contained in $(\mathbf{X}) k[\mathbf{X}]$ intersect in zero, there exists such a prime ideal $P_{n-1}$ of $k[\mathbf{X}]$ such that $P_{n-1} \cap R \subsetneq(\mathbf{X}) k[\mathbf{X}] \cap R$. Repeating this argument, we find a strictly descending chain of prime ideals contained in $R$ : $(\mathbf{X}) k[\mathbf{X}] \cap R \supsetneq\left(P_{n-1} \cap R\right) \supsetneq \cdots \supsetneq P_{0}=(0)$.

This behavior where the dimension of the homogeneous spectrum of a graded integral domain $R$ is less than $\operatorname{dim} R$ also occurs in the case where $R$ is an $\mathbb{N}$-graded integral domain. For example, if $A$ is a one-dimensional quasilocal integral domain 
such that the polynomial ring $A[X]$ has dimension three [9, then the homogeneous spectrum of $A[X]$ in its natural $\mathbb{N}$-grading has dimension two.

\section{REFERENCES}

[1] D. Eisenbud, Commutative Algebra with a view toward Algebraic Geometry, Springer-Verlag, New York, 1995. MR 97a:13001

[2] R. Gilmer, Commutative Semigroup Rings, University of Chicago Press, Chicago, 1984. MR 85e: 13018

[3] I. Kaplansky, Commutative Rings, Allyn and Bacon, Boston, 1970. MR 40:7234

[4] E. Kunz, Introduction to Commutative Algebra and Algebraic Geometry, Birkhäuser, Boston, 1985. MR 86e: 14001

[5] H Matsumura, Commutative Ring Theory, Cambridge University Press, Cambridge, 1986. MR 88h:13001

[6] S. Mori, Über eindeutige Reduktion von Idealen in Ringen ohne Teilerkettensatz, J. Sci. Hiroshima Univ. Ser. A 3(1933), 275-318.

[7] M. Nagata, Local Rings, Interscience, New York, 1962. MR 27:5790

[8] J. Ohm and R. Pendleton, Rings with Noetherian spectrum, Duke Math. J., 35 (1968), 631639. MR 37:5201

[9] A. Seidenberg A note on the dimension theory of rings, Pac. J. Math., 3 (1953), 505-512. MR 14:941c

[10] O. Zariski and P. Samuel, Commutative Algebra, volume I, Van Nostrand, New York, 1958. MR 19:833e

Department of Mathematics, Purdue University, West Lafayette, Indiana 47907-1395

E-mail address: heinzer@math.purdue.edu

Department of Mathematics, University of Haifa, Mount Carmel, Haifa 31905, Israel

E-mail address: mroitman@math.haifa.ac.il 